\title{
Chronic Electrical Stimulation of the Intact Corticospinal System after Unilateral Injury Restores Skilled Locomotor Control and Promotes Spinal Axon Outgrowth
}

\author{
Jason B. Carmel, ${ }^{1}$ Lauren J. Berrol, ${ }^{2}$ Marcel Brus-Ramer, ${ }^{2}$ and John H. Martin ${ }^{2,3,4,5,6}$ \\ Departments of ${ }^{1}$ Neurology, ${ }^{2}$ Neuroscience, ${ }^{3}$ Neurological Surgery, and ${ }^{4}$ Psychiatry, Columbia University, New York, New York $10032,{ }^{5}$ New York State \\ Psychiatric Institute, New York, New York 10032, and ' Department of Physiology, Pharmacology, and Neuroscience, City College of the City University of \\ New York, New York 10031
}

Injury to the brain or spinal cord usually preserves some corticospinal (CS) connections. These residual circuits sprout spontaneously and in response to activity-based treatments. We hypothesized that augmenting activity in spared CS circuits would restore the skilled motor control lost after injury and augment outgrowth of CS terminations in the spinal cord. After selective injury of one half of the CS tract (CST) in the rat, we applied $10 \mathrm{~d}$ of electrical stimulation to the forelimb area of motor cortex of the spared half and tested motor performance for $30 \mathrm{~d}$. Rats with injury and CST stimulation showed substantial improvements in skilled paw placement while walking over a horizontal ladder. By the end of the testing period, the walking errors of the previously impaired forelimb in rats with injury and stimulation returned to baseline, while the errors remained elevated in rats with injury only. Whereas the time to perform the task returned to normal in all animals, the pattern of errors returned to normal only in the stimulated group. Electrical stimulation also caused robust outgrowth of CST axon terminations in the ipsilateral spinal cord, the side of impairment, compared with rats with injury only. The outgrowth was directed to the normal gray matter territory of ipsilateral CST axon terminations. Thus, stimulation of spared CS circuits induced substantial axon outgrowth to the largely denervated side of the spinal cord and restored normal motor control in the previously impaired limbs.

\section{Introduction}

The corticospinal (CS) system is the principal system for skilled voluntary movement in humans (Porter and Lemon, 1993). Damage to the CS tract (CST) accounts for much of the movement impairment caused by brain or spinal cord injury (Bareyre et al., 2004; Thomas et al., 2005; Lindenberg et al., 2010). After injury, recovery of motor function, both spontaneous (Weidner et al., 2001) and with therapy (Schwab and Brösamle, 1997; Bradbury et al., 2002; Dergham et al., 2002; Tuszynski et al., 2003; Freund et al., 2006; Maier et al., 2008), is attributed to CST repair.

Activity is critical for normal formation and function of the developing CS system (Martin et al., 2009). Electrical stimulation of the CST biases developing CST axons, favoring stimulated axons at the expense of nonstimulated axons, through activitydependent competition (Salimi and Martin, 2004). Kittens with

\footnotetext{
Received March 19, 2010; revised June 25, 2010; accepted June 29, 2010.

This work was supported by National Institutes of Health Grants K12NS001698 (J.B.C.), R01NS64004 (J.H.M.), TL1RR024158 (J.B.C.), and GM07367 (M.B.-R.), and the New York State Spinal Cord Injury Research Board Grant C022064 (J.B.C. and J.H.M.). We thank Xiuli Wu for histochemistry and histology and Dr. Kathleen Friel for help with Matlab.

Correspondence should be addressed to either of the following: Dr. Jason B. Carmel, Department of Neurology, Columbia College of Physicians and Surgeons, 180 Fort Washington Avenue, New York, NY 10032, E-mail: jbc28@columbia.edu; or Dr. John H. Martin, Department of Physiology, Pharmacology, and Neuroscience, City College of the City University of New York, 160 Convent Avenue, New York, NY 10031, E-mail: jmartin@ccny.cuny.edu.

DOI:10.1523/JNEUROSCI.1435-10.2010

Copyright $\odot 2010$ the authors $\quad 0270-6474 / 10 / 3010918-09 \$ 15.00 / 0$
}

primary motor cortex (M1) inactivation or restricted forelimb use do not develop normal CST connections and demonstrate lasting motor deficits (Martin et al., 2004). Balancing activity levels from each half of the CS system restores spinal innervation and skilled motor control (Friel and Martin, 2007).

We previously used unilateral injury or stimulation of the rat CST to study injury- and activity-dependent plasticity in maturity (Brus-Ramer et al., 2007). The rat CST, like the human, is largely crossed, and unilateral injury leaves sparse CS innervation to the spinal cord on the impaired side (Brösamle and Schwab, 1997). Unilateral injury or electrical stimulation causes outgrowth and strengthening of the sparse CST ipsilateral to the intact half of the CS system or ipsilateral to stimulation (BrusRamer et al., 2007). Importantly, electrical stimulation of the intact CS system created the most robust CST spinal connections, with an approximately additive effect of injury and activity.

Here we ask whether electrical stimulation of the intact half of the CS system after unilateral injury, to promote ipsilateral CST connections, can help restore skilled motor function. M1 ipsilateral to the impaired side is well suited to restore CS control to the impaired limbs. The ipsilateral forelimb motor representation is similar to that of the contralateral representation (Brus-Ramer et al., 2009). Also, the ipsilateral and contralateral CST spinal terminations target similar premotor circuits in the intermediate laminae (Brus-Ramer et al., 2007). Finally, in humans with unilateral injury, one hemisphere can exert control over both hands (Werhahn et al., 2003), particularly after injury early in develop- 


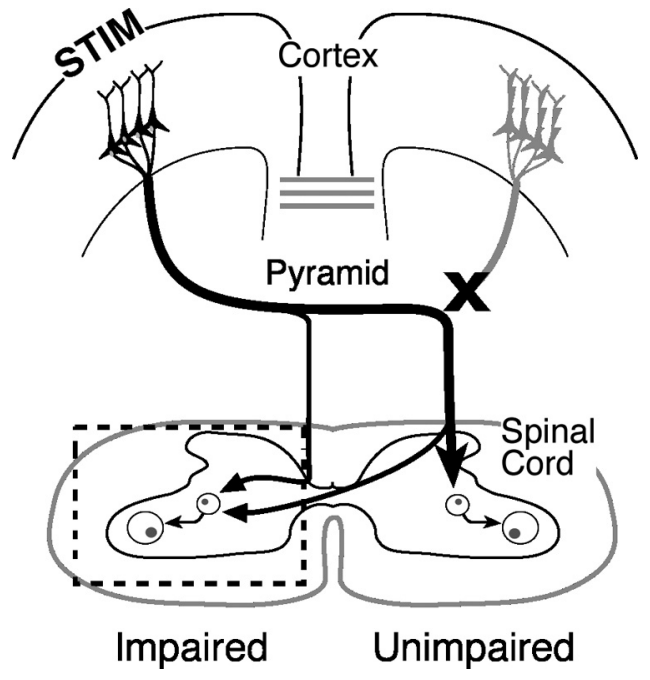

Figure 1. Schematic of experimental methods. All rats were subjected to unilateral pyramidotomy ( $" X$ "). The day after injury, the intact half of the $(S$ system was electrically stimulated (STIM) daily for $10 \mathrm{~d}$. We measured CS axon length in the impaired half of the spinal cord (dashed box).

ment (Lotze et al., 2009). Although we studied spared ipsilateral connections to spinal motor circuits after injury and stimulation, we propose that this also models the response of sparse contralateral CST axons spared after incomplete injury.

After unilateral injury of the CST, we stimulated M1 on the intact side daily for $10 \mathrm{~d}$. Stimulated rats demonstrated full recovery of motor deficits that extended beyond the stimulation period. The restoration of function was accompanied by robust outgrowth of ipsilateral CST axon terminations to the impaired side of the spinal cord. Thus, we demonstrate that CST electrical stimulation restored normal motor control and promoted dense CS innervation to impaired limbs.

\section{Materials and Methods}

Overview. We tested whether electrical stimulation of the intact half of CST after unilateral injury could restore skilled motor function (Fig. 1). We trained rats to walk over a horizontal ladder until a stable baseline error rate was established. We implanted an electrode over the forelimb motor cortex before cutting the pyramid opposite to the electrode in all rats. We used the cortical electrode to deliver electrical stimulation for $6 \mathrm{~h}$ daily over $10 \mathrm{~d}$ in the rats with injury and stimulation $(n=5)$ but not rats with injury only $(n=5)$. We measured performance of walking over a horizontal ladder at days 5, 10,15,20,25, and 30. In addition, we measured the time to cross the ladder and the types of errors made with the impaired forelimb. On day 31 or 32 after injury, the motor cortex of the intact CST was injected with biotinylated dextran amine (BDA) to anterogradely label the CST. Twelve days later, rats were killed and the cervical spinal cord cut in cross section. CST axon terminations in the spinal cord on the impaired side of the rats (Fig. 1, dashed box) were visualized and measured using a stereological estimate of axon length. We compared motor performance and the density and distribution of axon terminations between rats with injury and stimulation and rats with injury only. We also used stimulation of the CST at the pyramid ( $n=2$ injury and stimulation and $n=2$ injury only) to ensure that the CST was stimulated selectively.

Surgical. Experiments were conducted on adult female Sprague Dawley rats (wt 225-275 g). The surgeries (electrode implantation, pyramid lesion, and CST axon tracing) were performed under general anesthesia ( $80 \mathrm{mg} / \mathrm{kg}$ ketamine; $10 \mathrm{mg} / \mathrm{kg}$ xylazine, via intraperitoneal injection) and sterile conditions. All procedures were approved by the institutional animal care and use committees of Columbia University, the
New York State Psychiatric Institute, and City College of the City University of New York.

Electrode implantation and stimulation. Both injury-only and injuryplus-stimulation rats received the same electrode implantation; only the stimulated animals received stimulation. Briefly, rats were anesthetized and placed in a stereotactic frame (Kopf Instruments). We made a craniotomy over the forelimb area of motor cortex, as in our previous study (Brus-Ramer et al., 2009). Two stainless steel stimulating electrodes (0.005 inch insulated stainless steel; Plastics One) were placed over the dura. The wires were bent into an "L" shape with the bottom of the L deinsulated. Wires were run parallel $1.5 \mathrm{~mm}$ apart and $4 \mathrm{~mm}$ long. We placed the angle of the L-shaped electrodes $2 \mathrm{~mm}$ and $3.5 \mathrm{~mm}$ lateral to bregma, respectively, with the exposed contact extending rostrally over the forelimb motor cortex.

To confirm proper placement of the electrodes, we stimulated motor cortex to provoke a motor response. A constant current stimulator (A-M Systems) was used to deliver trains of stimuli $(0.2 \mathrm{~ms}$ duration, $333 \mathrm{~Hz}$, $45 \mathrm{~ms}$, every $2 \mathrm{~s}$ ). We adjusted the current to the minimal value to evoke contralateral forelimb movements, which was typically 1.1-1.8 mA. Once proper placement was confirmed, we secured the electrodes using microscrews (Small Parts) and dental acrylic cement. The wires were attached to a connector at the top of the head that was secured to the skull with screws and dental acrylic cement. We placed stimulating electrodes at least 1 week before CST injury and determined that electrode placement caused no change in motor performance (data not shown). Pyramid electrode placement was performed as previously described (BrusRamer et al., 2007).

Beginning the day after pyramidotomy, we stimulated rats $6 \mathrm{~h}$ daily for $10 \mathrm{~d}$. For electrical stimulation, the connector on the rats' heads was attached to a commutator (Plastics One) mounted to the top of the cage, and trains of stimuli (described above) were given at the threshold to provoke a contralateral forelimb motor response. The motor response was limited to the forelimb contralateral to stimulation; we never observed hindlimb or ipsilateral forelimb movements. Rats were given stimulation at the initial motor threshold for the entire $6 \mathrm{~h}$ period, although the motor responses usually disappeared soon after initiating stimulation. Rats ate, drank, and slept during the stimulation. They never pawed at their heads or showed any signs of pain or distress. On days when both behavior testing and stimulation were performed (days 5 and 10), behavior testing was done first.

Pyramidotomy. All rats had complete unilateral lesion of one CST, as described previously (Brus-Ramer et al., 2007). We injured the pyramid contralateral to the preferred forelimb, to maximize the motor impairment because motor deficits after pyramidotomy in rats are subtle (Whishaw et al., 1993). Forelimb preference was determined using a pasta manipulation task (Allred et al., 2008). For pyramidotomy, rats were placed supine in head fixation and the pyramids exposed. One pyramid was completely transected at the rostral medulla using iridectomy scissors and a second pass with a microknife. Lesions were confirmed to be histologically complete without extension into adjacent structures using Kluver-Barrera staining (Kluver and Barrera, 1953) or dark-field microscopy of the medulla at the site of injury (see supplemental Fig. 1, available at www.jneurosci.org as supplemental material).

Horizontal ladder walking. Rats were trained and tested on a horizontal ladder with Plexiglas sides, a starting platform, and an end platform that leads to their home cage, which is similar to several prior studies (Metz and Whishaw, 2002; Maier et al., 2008). We trained rats at least three times a week until they crossed the ladder without hesitation and demonstrated a stable forelimb error rate below $20 \%$. The total number of runs across the ladder during the training phase was $\sim 300$ for each rat. Rats were motivated to cross the ladder by placing their home cage at the end. We further motivated rats that hesitated initially during training with an air puff delivered at the entry of the ladder. Testing sessions were performed at the same time of day. Baseline error rates were established with at least two testing sessions within $5 \mathrm{~d}$ of pyramidotomy. Testing was performed every $5 \mathrm{~d}$ after injury without additional training in between. During testing sessions, rats were run for 10 trials in each direction on the ladder. Rung position was changed during the session to prevent rats from adapting to a particular pattern. The time to cross the ladder was 
recorded only for runs in which the rat stayed in constant motion from start to end. Given that the time to cross the ladder averages $\sim 2 \mathrm{~s}$, rats received only $40 \mathrm{~s}$ worth of testing every $5 \mathrm{~d}$ after pyramidotomy. The number of runs given over the total $30 \mathrm{~d}$ testing period (120) was much smaller than was given during training (300), and was given less frequently (every fifth day rather than every day or every other day). We believe this limited amount of activity associated with testing was unlikely to drive activity-dependent plasticity on its own.

All trials were video recorded using a consumer digital video camcorder (Canon ZR960) at $30 \mathrm{~Hz}$ with $1200 \mathrm{~W}$ of illumination. Videos were analyzed frame by frame. Paw placement was measured while the rat was in motion. If the rat stopped, no measurements were made for the step before the stop and at least two steps after the stop. Steps with placement of the palm of the forepaw, between the wrist and the digits, on the rung were scored as good (Metz and Whishaw, 2002)). All other steps were recorded as errant. For the impaired forelimb, we categorized the errors into one of three types: oversteps (the paw contacts the rung at or proximal to the wrist), understeps (the paw contacts the rung with the digits), and missed steps (the paw did not contact the rung). The overall error rate is the number of errant steps over the total number of steps. The error rate for each error type is the number of errant steps (oversteps, understeps, or misses) over the total number of steps. Thus adding the error rates for each error type equals the overall error rate.

Anterograde labeling of CST axons. On day 31 or 32 after injury, we injected BDA into the forelimb area of motor cortex to anterogradely label the intact CST, as in our previous study (Brus-Ramer et al., 2007). Briefly, after inducing anesthesia, we removed the stimulating electrode and exposed the cortical surface. For either animal group, we made 15 injections (300 $\mathrm{nl}$ each) to densely label the intact CST. After a $12 \mathrm{~d}$ survival, rats were given an anesthetic overdose and perfused through the heart with saline followed by $4 \%$ paraformaldehyde. The brain and spinal cord were removed, postfixed in the same fixative at room temperature for $2 \mathrm{~h}$, and then transferred to $20 \%$ sucrose in $0.1 \mathrm{~m}$ phosphate buffer at $4^{\circ} \mathrm{C}$ overnight. Transverse sections of the sixth cervical section were cut at $40 \mu \mathrm{m}$ and processed for BDA. For visualization of BDA, sections were incubated in PBS, pH 7.4, containing $1 \%$ avidin-biotin complex reagent (ABC kit; Vector Laboratories) and $0.2 \%$ Triton X-100 for $2 \mathrm{~h}$ at room temperature. After rinsing, sections were incubated with the chromogen diaminobenzidine (DAB; Sigma) for 6-30 min. After rinsing again, sections were mounted on gelatin-coated slides, air dried overnight, dehydrated, and coverslipped. We also collected sections through the pyramid, to verify a complete lesion that did not intrude into adjacent structures (see supplemental Fig. 1, available at www.jneurosci.org as supplemental material).

Axon length estimation. Axon length was measured using the "space balls" probe in the Stereo Investigator stereology program (MBF Bioscience) (Mouton et al., 2002). We chose this method because it allows accurate and efficient estimates of axon length. It can also be used to estimate axon length in tissue with high or low axon density, allowing comparisons of CST innervation of various motor centers. Finally, if similar sampling and normalization is used by different investigators, the amount of CST innervation could be compared across studies and levels of the neuraxis, making it an effective in vivo growth assay. After we traced the area of interest, which was the spinal cord gray matter ipsilateral to BDA tracing (Fig. 1, dashed box), the program places virtual spheres ("space balls") within the tissue. The user defines the size of the sphere to be used and the area in which the sphere should be placed (the counting frame). Together, these determine the density of sampling. Because labeled CST terminations ipsilateral to BDA are sparse, we used relatively dense sampling parameters: a $20-\mu \mathrm{m}$-diameter sphere and square counting frames $50 \mu \mathrm{m}$ on each side, resulting in 437-577 sampling sites per section. At $1000 \times$ magnification (oil immersion), we marked intersections between the virtual sphere and labeled axons within the gray matter. An intersection is contact or crossing of the linear axon and the cross section of the sphere, a circle of varying diameter. The number of intersections (range 5-79) and the volume of the tissue sampled (area of gray matter $\times 40 \mu \mathrm{m}$ thickness) were used to estimate axon length (Mouton et al., 2002). Axon length estimates were made for five or six sections for each animal and the lengths averaged. To validate this technique, in two rats we traced all of the axons within the same sections used for the stereological analysis, using our published methods (BrusRamer et al., 2007). For these rats, the estimates by stereological sampling were within $10 \%$ of the lengths determined by tracing all of the labeled axons (data not shown).

Our axon length estimates were corrected for the variability in tracing between animals using the average number of BDA-labeled axons within the main CST. First, the number of BDA-labeled axons in the base of the dorsal column opposite cortical injection was estimated using the Optical Dissector program in Stereo Investigator. The estimated number of dorsal column axons for each of the sections was averaged per rat. The number for each rat was then divided by the average for all rats in this study to generate the correction factor. We found that this correction factor produced results roughly equivalent to using the number of labeled axons within the ipsilateral CST, as in our previous study (BrusRamer et al., 2007), and gave less variability (data not shown).

Regional axon length analysis. We represented changes in axon length within the gray matter using density maps and a line graph of density along the dorsoventral axis. The contour of the gray matter and intersections between the virtual spheres and the labeled axons in the spinal cord ipsilateral to injection were used to create a TIFF file with intersections represented by a single pixel. Individual sections were corrected for orientation and aligned with one another according to fiduciary marks before being exported and quantified using custom programs written in Matlab (MathWorks). Output files of individual sections of the spinal gray matter with labeled interactions were divided into 52 by $52 \mu \mathrm{m}$ square regions of interest (ROIs). For each ROI, we used the number of interactions to compute an associated axon length. Length is represented according to a color scale, from the lowest density (blue) to the highest (red) with the associated numbers indicating an estimate of true axon length. Areas without labeling are coded white. We generated regional density maps for individual animals and then averaged for animals within each treatment group. We used the same data to construct a dorsoventral plot using a custom program within Matlab, as previously described (Brus-Ramer et al., 2007). As with the heat maps, the scale for the dorsoventral plot represents a precise estimate of labeled axon length.

Statistics. Statistical analyses were performed using Prism 5 (Graph Pad) and Excel (Microsoft). The differences between groups over time were performed using repeated-measures ANOVA with a Bonferroni post hoc correction for testing individual time points. For the injury-andstimulation group, the same five rats were used for both behavioral and anatomical studies. For the injury-only group, two rats in the behavior study were not part of the anatomical analysis. One of these rats had poor BDA labeling, and the other had adhesion of the dura to the pia underlying the electrodes, which did not permit BDA labeling at all. Two other rats were used. These rats had equivalent conditions as the other rats, including the same behavior training and testing. These two rats were excluded from the behavioral analysis because each had baseline error rates above criterion (20\%).

\section{Results}

\section{CST electrical stimulation restores skilled locomotion}

Rats had unilateral lesion of one pyramid followed by epidural electrical stimulation of the forelimb M1 on the opposite (intact) hemisphere (Fig. 1). We tested motor performance in rats with injury and stimulation $(n=5)$ and rats with injury only $(n=5)$ using a horizontal ladder with irregularly spaced rungs. Errors of paw placement on the rungs were measured at baseline before pyramidotomy and every $5 \mathrm{~d}$ until day 30 . We predicted that there would be a large effect of stimulation in the impaired forelimb, because stimulation was performed over the forelimb area of $\mathrm{M} 1$.

Indeed, the affected forelimb showed improvement in rats with injury and M1 stimulation versus rats with injury only (Fig. $2 A$ ) (repeated-measures ANOVA, $F=35.89, p<0.0001$ ). Both at baseline and at day 5 after injury, the error rate of the injury with stimulation group was not different from the injury-only 
A Impaired Forelimb

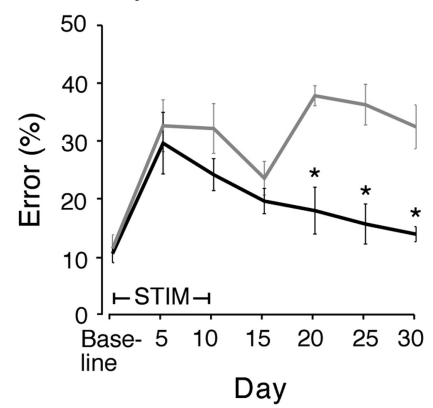

C Impaired Hind Limb

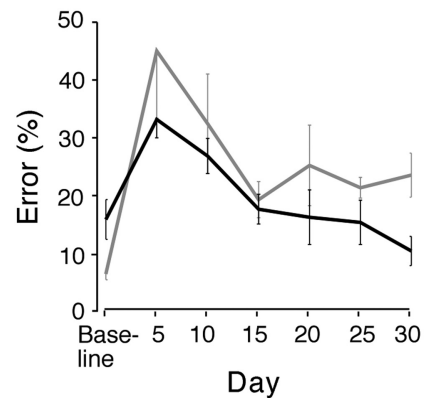

B Unimpaired Forelimb

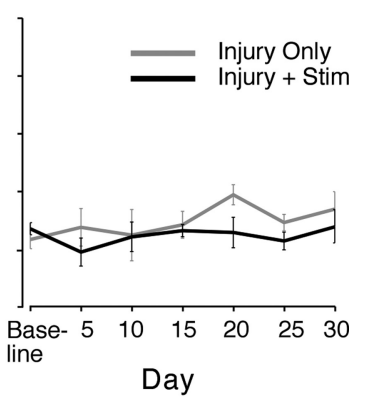

D Unimpaired Hind Limb

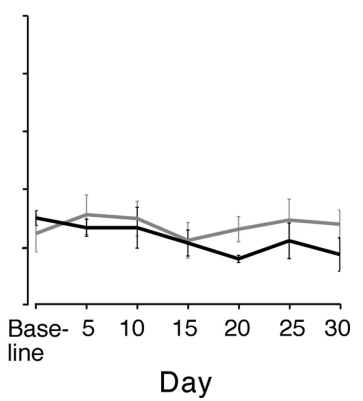

Figure 2. Motor cortex electrical stimulation improves ladder-walking performance. Rats were trained to cross a horizontal ladder with irregularly spaced rungs. Error rates were measured at baseline and every $5 \mathrm{~d}$ after injury to day 30 . Mean error rates at each testing time are shown \pm SEM (except day 5 in the impaired hindlimb of injury-only rats shows only - SEM). $\boldsymbol{A}$, Impaired forelimb. Error rates were not different at baseline or day 5 , but they differed overall $(F=35.89, p<0.0001$, repeated-measures ANOVA). By day 20, the error rates in the two groups were significantly different, and this persisted at days 25 and 30 ( $p<0.01$, asterisks). For the impaired hindlimb $(\boldsymbol{C})$, there was no significant improvement with forelimb motor cortex stimulation. The performance in the unimpaired limbs $(\boldsymbol{B}, \boldsymbol{D})$ did not change with either injury or stimulation.

group (Bonferroni post hoc correction of repeated-measures ANOVA, $p>0.05)$. The injury-only group had no decrease in errors of the affected forelimb from day 5 to day 30 ( $t$ test, $p=$ $0.98)$. For the injury-only group, the day 30 error rate was significantly higher than baseline $(p=0.006)$. The rats with injury and stimulation had a decline in forelimb errors from $5 \mathrm{~d}$ after injury to day 30 ( $p=0.019)$. Importantly, the error rate at day 30 was not significantly different from baseline $(p=0.13)$. Rats with M1 stimulation had a reduction in the affected forelimb error rates at day 20 after injury, $10 \mathrm{~d}$ after the end of stimulation. Compared with rats with injury only, this difference was highly significant (day 20, $p<0.001$ ). The difference between rats with injury only and rats with injury and stimulation continued to be significant at day $25(p<0.001)$ and day $30(p<0.01)$ after injury. Thus, M1 stimulation over the forelimb representation completely ameliorated the forelimb deficits caused by unilateral CST injury. The effects of stimulation persisted as long as they were tested, out to $30 \mathrm{~d}$.

We also tested the effect of cortical stimulation for $10 \mathrm{~d}$ over the forelimb area on the impaired hindlimb performance (Fig. $2 C)$. At baseline, rats in the injury-and-stimulation group had slightly higher error rates than those in the injury-only group $(p=0.03)$, but there was no difference at day $5(p=0.35)$. Both groups improved between days 5 and 15 , and the rats receiving stimulation continued to have a decline in error rate out to $30 \mathrm{~d}$. However, in contrast to forelimb motor recovery, the effect of stimulation on hindlimb motor recovery over the $30 \mathrm{~d}$ was not

significantly different from the injury-only group (repeatedmeasures ANOVA, $F=3.86, p=0.06$ ). Also, restoration of skilled forelimb control was specifically related to M1 stimulation and not to a generalized trend in improved performance during the testing period. We found no significant change in error rate in the unimpaired forelimb (Fig. $2 B$ ) (ANOVA, $F=0.92, p=0.48$ ) and unimpaired hindlimb (Fig. $2 D)(F=0.71, p=0.64)$. These findings indicate that electrical stimulation over the forelimb M1 caused selective restoration of motor function in the impaired forelimb.

To test whether the effects of electrical stimulation are mediated locally within M1 or are due to activity of the whole CS system, we stimulated the intact pyramid in a small number of rats with injury to the other pyramid ( $n=2$ stimulation, $n=2$ controls). Pyramid stimulation causes selective activation of the CST (Brus-Ramer et al., 2007). Like M1 stimulation, pyramid stimulation ameliorated forelimb deficits (supplemental Fig. 2, available at www.jneurosci.org as supplemental material), suggesting that the CST, rather than other motor circuits, mediates the behavioral effects of stimulation.

\section{M1 electrical stimulation ameliorates all types of forelimb misplacements}

CST injury can cause specific deficits in forelimb endpoint control. In humans, CST injury is often accompanied by significant undershooting of the target during reaching (Sukal et al., 2007), whereas cats with CS system defects overstep the rungs of a horizontal ladder (Friel et al., 2007) and overshoot the target during reaching (Martin et al., 2000). For the affected forelimb, we determined the separate types of targeting errors: oversteps, understeps, and missed steps. Missed steps are the most severe misplacement and likely represent a mix of very hypometric steps to the next distal rung and very hypermetric steps to the next proximal rung. We first determined whether injury only produced hypometria, hypermetria, or a generalized loss of skilled placement. We then examined whether stimulation restored the different error classes equally. If one or another error type remained elevated, then the animal might merely be compensating for a persistent defect.

Figure 3 presents the three types of errors in rats with injury only and rats with injury and stimulation. Error rates are presented for baseline and days 5-10, 15-20, and 25-30 to ease comparison of the two groups. Oversteps comprised the majority of errors at baseline in both groups of rats (injury only, 79\%; injury + stim, $81 \%$ ). Injury produced increases in each type of error to similar degrees, and the error were also similar for rats with injury only versus rats with injury and stimulation (oversteps $277 \%$ vs $237 \%$; understeps $270 \%$ vs $307 \%$; missed steps $362 \%$ vs $355 \%$ ). Electrical stimulation caused reduction in all types of errors by the end of the testing period. For oversteps (Fig. 3A), the overall difference between the groups was highly significant $(F=12, p=$ 0.002, repeated-measures ANOVA). Post hoc testing revealed a significant difference at days 25-30 ( $p<0.05)$, and oversteps were reduced by $53 \%$ compared with controls. The understeps (Fig. 3B) and missed steps (Fig. 3C) did not have significant differences overall with ANOVA testing, due to the small numbers of each of these error types. However, at days 25-30, understeps were reduced by $80 \%$ and misses by $39 \%$ compared with controls. Thus, pyramidotomy produces a similar increase in all error classes, indicating a generalized loss of placement accuracy. Although only oversteps were significant, stimulation ameliorated each of these deficits to similar degrees. Our results suggest that 


\section{A Oversteps}

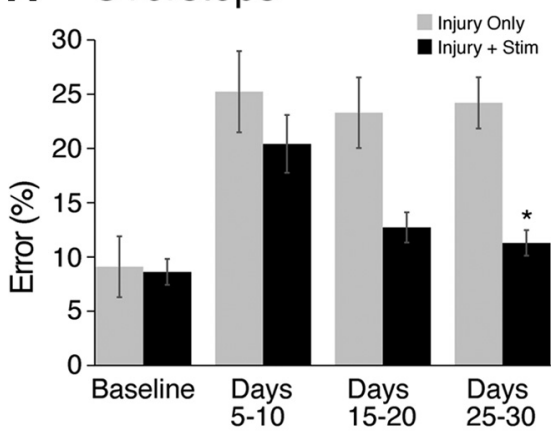

\section{B Under Steps}

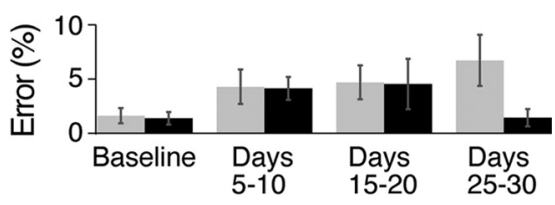

\section{Misses}

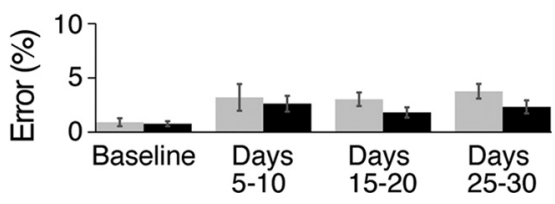

Figure 3. Electrical stimulation improves all classes of forelimb paw placement errors. Rats make several types of errors while walking on a horizontal ladder (oversteps, understeps, or missed steps; see Materials and Methods), with the majority being oversteps. We compared the error rates at baseline, days 5-10, days $15-20$, and days $25-30$. $A$, Oversteps. The overall difference between the groups was highly significant $(F=12, p=0.002$, repeated-measures ANOVA). Post hoc testing revealed a significant difference at days $25-30$ ( $p<0.05$, asterisk). $\boldsymbol{B}, \boldsymbol{C}$, The understeps $(\boldsymbol{B})$ and missed steps $(\boldsymbol{C})$ did not have significant differences overall with ANOVA testing, due to the small numbers of each of these error types. However, at days $25-30$, oversteps were reduced by $53 \%$, understeps by $80 \%$, and misses by $39 \%$ compared with injury only, suggesting reduction of all error types with stimulation.

stimulation restores normal motor control rather than producing a compensatory strategy.

Rats with injury and stimulation cross the ladder as quickly as rats with injury only

We wanted to ensure that the improvement in skilled locomotion was due to improved motor control, as opposed to a more cautious and slower walking strategy (Fitts, 1954). For each rat, we measured the time for each ladder crossing and averaged the individual trial values for each testing day. We used the mean time to cross and the mean forelimb error rate for each testing day to create a unique point for each rat. Figure 4 compares the results at baseline and at the times that show improvement in the stimulated rats-days 20-30. Points are colored according to the legend. The mean time and error values ( \pm SEM) were used to create the plus $(+)$ symbols, which are colored the same as the points used to create these mean values. At baseline, the time to cross was not different for the injury-and-stimulation rats and the injury-only rats $(p=0.22)$. The error rates were also not different, as discussed above. Injury only caused the rats to have many more errors, but the time to cross did not increase. Rats with stimulation could achieve lower error rates after injury by adopting a more cautious strategy (points shifted down and to right) or by reducing errors without crossing the ladder more slowly (points shifted down but not to right). At the time of

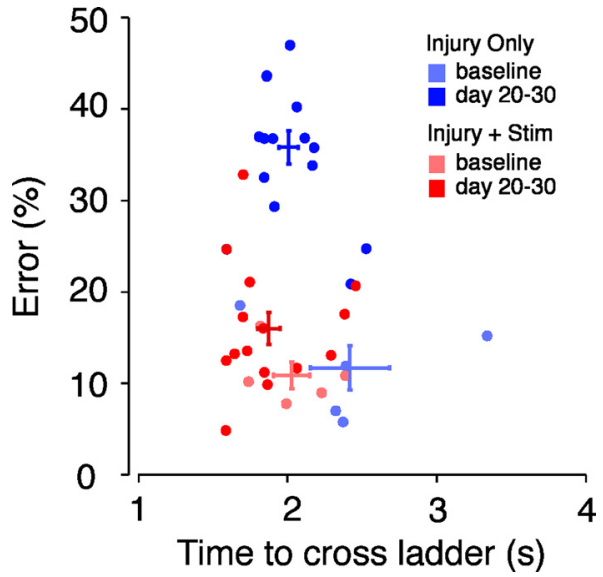

Figure 4. Rats with injury and stimulation cross the ladder as quickly as rats with injury only. The relationship between speed and accuracy is demonstrated by plotting the forelimb error rate against the time to cross the ladder. The dots plot the error rate and time to cross for a single rat on one day of testing. We compared baseline against the time of improvement, days $20-30$. The points are colored according to the legend. The mean time and error values ( \pm SEM) were used to create the plus $(+)$ symbols, which are colored the same as the points used to create these values. Rats with injury only and rats with injury and stimulation did not differ in the time to cross the ladder either at baseline ( $p=0.22)$ or at days $20-30(p=0.1)$.

behavior improvement, the groups also did not differ in the time to cross the ladder $(p=0.1)$. This occurred despite the error rate being lower in the injury-and-stimulation group than in the injury-only group. This implies that recovery of motor control, rather than a more cautious strategy, underlies the recovery of function in the stimulated animals.

\section{Electrical stimulation promotes outgrowth of CST axon} terminations to the spinal cord on the impaired side

Stimulation of the intact pyramid promotes CST outgrowth into the cervical spinal cord (Brus-Ramer et al., 2007). Whereas stimulation of the intact M1 could promote CS system axon outgrowth, or strengthen connections, at multiple levels, we wanted to determine whether it also promoted spinal outgrowth at the C6 level, as pyramid stimulation does. Within $2 \mathrm{~d}$ of the last behavior testing session (day 31 or 32 after injury), BDA was injected into the forelimb motor cortex, as in our previous study (Brus-Ramer et al., 2007). Rats were killed, and the C6 segment of spinal cord cut transversely and stained for BDA. BDA-labeled CST axon terminations were sampled using a stereological method for length assessment. The axon length was then normalized to the labeling efficiency of each rat (see Materials and Methods for full description).

The results are pictured in Figure 5. For both the photomicrographs (top row), and the heat maps (middle row), the images on the left (Fig. 5A) are of injury-only rats and the images on the right (Fig. $5 B$ ) injury and stimulation; outlined by a black line is the spinal cord gray mater ipsilateral to stimulation (e.g., dashed box in Fig. 1). The photomicrograph of the representative rat with injury only (Fig. 5A1; inset shows location) shows much sparser BDA-labeled axon terminations within the gray matter than the representative rat with injury and stimulation (Fig. 5B1). For the heat maps, each $52 \mu \mathrm{m}$ by $52 \mu \mathrm{m}$ region of interest is colored according to the length of labeled axons in each, and the normalized axon length per section is indicated by the color bar at the right. Representative rats (Fig. 5A2,B2) and the average axon label for each group (Fig. 5A3,B3) are shown for each group. There is a substantial increase in the density of axon label in the 


\section{A Injury Only}

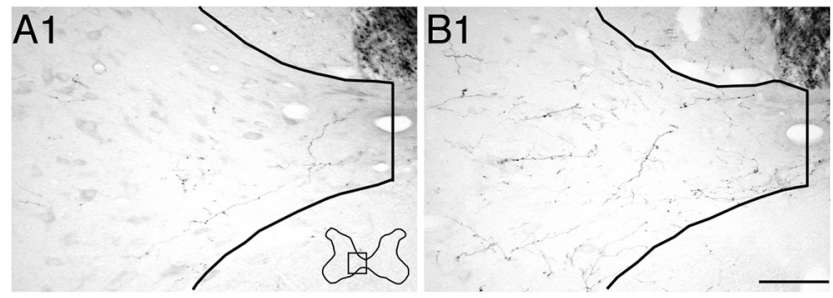

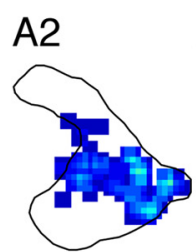

Individual

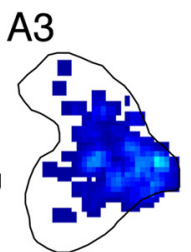

Group

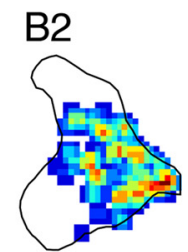

Individual

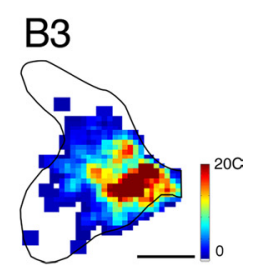

Group

\section{Axon Length}

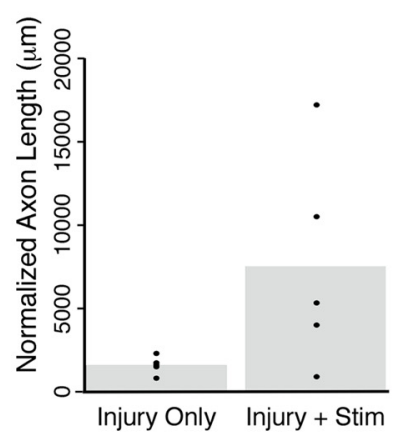

D Dorsoventral Plot

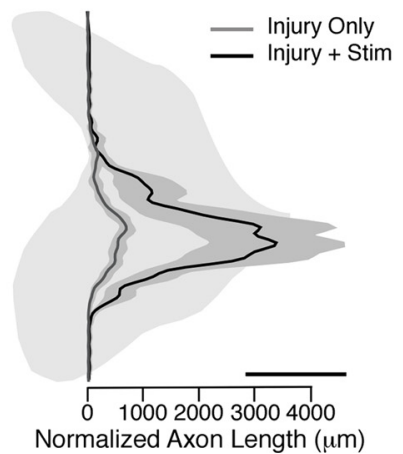

Figure 5. M1 stimulation promotes $C S$ axon outgrowth to the spinal cord on the impaired side of the rat (i.e., Fig. 1, dashed box). $\boldsymbol{A}$, Injury-only rats. $\boldsymbol{B}$, Injury-and-stimulation rats. The top row contains photomicrographs of $(6$ spinal cord cut in transverse section and stained for BDA. Inset in $\boldsymbol{A} \mathbf{1}$ shows the approximate location of the pictures. Midline was determined by bisecting the central canal. Magnification, $200 \times$; scale bar, $100 \mu \mathrm{m}$. The gray matter ipsilateral to BDA injection is outlined and has sparse BDA-labeled axons in the representative rat with injury only $(\boldsymbol{A} \mathbf{1})$, while the rat with injury and M1 electrical stimulation (B1) has more BDAlabeled axons. The heat maps (middle row) demonstrate axon length as a density map colored from blue (low) to red (high). The color bar demonstrates the axon length in micrometers within each region of interest. A representative rat and the average data for rats $(n=5)$ in each group are pictured. Stimulation caused abundant local outgrowth, which is densest in medial lamina 7. Scale bar, $500 \mu \mathrm{m}$. C, Quantification of axon length within the gray matter ipsilateral to stimulation. Bars plot mean axon length for each group; dots represent individual rats. $\boldsymbol{D}$, Dorsoventral plot of axon label. The same data used to generate the heat maps were used to create this dorsoventral plot of axon label. Stimulation caused robust outgrowth without spread beyond the dorsoventral distribution of rats with injury only. Scale bar, $500 \mu \mathrm{m}$.

injury-and-stimulation rats compared to the rats receiving injury only. The densest area of axon label for both groups of rats is the medial portion of lamina 7 with gradual decreases in density lateral, ventral, and dorsal to the hotspot. While the axon label is significantly higher in rats with injury and stimulation, the distribution remains similar to rats with injury only, indicating that stimulation did not produce mistargeted outgrowth.

Mean axon length for each group is shown in Figure 5C, with data from individual rats represented by black dots. The injuryonly rats are tightly clustered about the mean value of $1605 \pm 239$ $\mu \mathrm{m}$ of axon length per $40 \mu \mathrm{m}$ section. The rats receiving injury and $10 \mathrm{~d}$ of motor cortex stimulation had $7540 \pm 2860 \mu \mathrm{m}$ of axon length per $40 \mu \mathrm{m}$ section. Thus, stimulation caused substantial outgrowth, equal to 4.7 times the mean axon length of the rats with injury only ( $t$ test, $p=0.03$ ). Whereas the stimulated group had a more variable distribution of axon length estimates, all but one was greater than the injury-only rats. Our findings indicate that M1 stimulation produced robust outgrowth of CST terminations in the spinal cord. Importantly, the axonal outgrowth assay was made $>30 \mathrm{~d}$ after cessation of M1 stimulation. This demonstrates persistence of stimulation-induced axonal outgrowth.

To determine whether stimulation caused outgrowth more strongly in one subregion of the gray matter, we plotted the estimated axon length for each group along the dorsoventral axis, as in our previous study (Brus-Ramer et al., 2007). The results, pictured in Figure $5 D$, are remarkably similar to the effect of pyramid stimulation (Brus-Ramer et al., 2007). The injury-only rats had the majority (62\%) of labeled axons within the motor laminae of the spinal cord, the intermediate zone, and ventral horn (ventral to the base of the dorsal columns). Stimulation augmented the amount of label proportionately and also had the majority (67\%) of labeled axons within the motor laminae of the spinal cord. This increase in outgrowth ventrally was significant $(p=0.010)$. Also significant was the outgrowth to the dorsal horn $(p=0.012)$. The growth in the dorsal horn was directed to the deep laminae, a normal distribution (Brus-Ramer et al., 2007), dominated by muscle receptor and cutaneous receptor terminations (Morris et al., 2004). Importantly, there was no outgrowth into the superficial dorsal horn, where nociceptive afferents terminate (Todd, 2002). Thus, electrical stimulation of the motor cortex produced robust outgrowth that was directed within the normal dorsoventral distribution of CST axon terminations.

\section{Discussion}

Electrical stimulation of the intact CST after unilateral injury can restore skilled motor function. The effect of M1 stimulation was to restore not just the overall error rate, but all three subclasses of forelimb errors as well, consistent with restoration of normal motor control, as opposed to a compensatory strategy. Indeed, on these measures, rats with injury and stimulation achieved proficiency of ladder walking indistinguishable from intact rats. Skilled motor recovery was associated with nearly a fivefold increase in ipsilateral CST axon terminations on the affected side of the spinal cord, with much of that increase occurring within the motor laminae of the spinal gray matter. Augmenting CST connectivity with the spinal cord provides an anatomical substrate for motor recovery. Both behavioral and anatomical effects of CST stimulation persist well beyond the $10 \mathrm{~d}$ of stimulation; the behavior on day 30 and anatomy on day $43-44$ were significantly improved in rats with stimulation.

\section{Behavioral recovery due to selective CS system electrical stimulation}

While other studies have ascribed motor recovery to repair of the CST, this is the first study that both selectively targets the CS system for an activity-based repair and demonstrates skilled motor recovery. Behavioral recovery was robust for CST stimulation of both the motor cortex and the pyramid. This suggests a conserved recovery mechanism, whether exogenous activity is supplied to the tract itself (pyramid stimulation) or close to the cell bodies (motor cortex stimulation). Likewise, our finding that each site of stimulation produced similar behavioral recovery increases the likelihood that it is activation of the CST, as opposed to other motor control systems, that produces the therapeutic effect. 
Cortical stimulation of the forelimb representation ameliorates motor deficits in the forelimb more strongly than the hindlimb, suggesting that outgrowth and motor improvement can be targeted depending on the area of motor cortex that is stimulated. The trend toward improvement in hindlimb motor control could be explained in several ways, including strengthening of intracortical connections between the two areas, and better coordination with an improved forelimb through intraspinal circuits (Rossignol et al., 1996).

\section{Timing and magnitude of CST axon outgrowth and relationship to motor recovery}

Behavioral recovery is accompanied by robust outgrowth of the CST on the impaired side. Our previous study using pyramid stimulation demonstrated that CST electrical stimulation drives activity-dependent growth and stronger connections to spinal motor circuits by the end of the $10 \mathrm{~d}$ stimulation period (BrusRamer et al., 2007). The magnitude of the outgrowth with M1 stimulation-4.7-fold that of rats with injury only-was much higher than we had seen in our previous study using pyramid stimulation (1.4-fold). There are two principal differences between the studies that might account for this difference: the site of stimulation and survival time. Pyramid stimulation activates CST axons selectively (Brus-Ramer et al., 2007). By contrast, M1 stimulation activates CST axons as well as axons that project to other motor centers, especially the red nucleus and reticular formation (Kuypers, 1981). M1 stimulation could elevate spinal excitability more than pyramidal stimulation, via indirect brainstem motor pathways. This could make the spinal cord a more receptive locus for functional CST outgrowth. In our previous study, rats with pyramid stimulation were killed at $10 \mathrm{~d}$ after CST injury, immediately after the stimulation period. In the present study, rats with M1 stimulation were killed at 43 or $44 \mathrm{~d}$ after CST injury. An intriguing possibility for further study is that electrical stimulation initiates a growth program within CS neurons that continues to promote axon outgrowth beyond the stimulation period. Thus, the more robust axon outgrowth seen in this study is likely due to greater outgrowth produced by M1 stimulation and/or the longer survival time and continued axon outgrowth.

The most remarkable finding of our study was the complete recovery of forelimb motor function in stimulated animals at $30 \mathrm{~d}$, with significant improvement beginning at $20 \mathrm{~d}$ after injury. Delayed recovery, observed after both M1 and PT stimulation, could reflect continued axon outgrowth after the stimulation period and more effective use of these new circuits in mediating motor recovery. We found a consistent initial improvement followed by worsening among the injury-only rats with M1 and PT electrodes (Fig. 2A; supplemental Fig. S2, available at www. jneurosci.org as supplemental material), suggesting that injurydependent sprouting of CST axons (Weidner et al., 2001; BrusRamer et al., 2007) was not sustained. Without further excitatory drive, in the absence of stimulation or limb use (Maier et al., 2008), spared ipsilateral CST terminations may be eliminated by activity-dependent competition for synaptic connections with spinal neurons. This possibility would also help to explain why axon outgrowth in this study was higher than our previous oneinitial axon outgrowth seen in injury-only rats of our previous study at $10 \mathrm{~d}$ may have regressed by $43 \mathrm{~d}$. Thus, CST stimulation may exert part of its effect by making injury-induced outgrowth more competitive.

\section{Relationship to other activity-based treatments and clinical application}

To restore function, we stimulated the intact half of the CS system after unilateral injury to drive growth to the ipsilateral spinal cord and create bilateral control from one half of the CS system. This approach contrasts markedly with other efforts to repair unilateral injury, which often attempt to increase excitability on the injured side or decrease excitability on the intact side. Such a rebalancing approach attempts to restore the normal CST wiring where each hemisphere controls the contralateral limbs. However, after severe unilateral injury, such as lesion of the pyramid, only one half of the CS system is available to restore motor control. We find that one half of the CS system is surprisingly adept at controlling movements on both sides of the body. We previously had shown similar representations of ipsilateral and contralateral movements within the forelimb area of motor cortex using an electrophysiological technique (Brus-Ramer et al., 2009). Now we demonstrate that electrical stimulation over the same area causes robust outgrowth of the CS terminations and behavioral improvement ipsilateral to stimulation. We had also shown that the ipsilateral motor actions of rat motor cortex depend on an intact CS system in the other hemisphere, and that this dependency can be eliminated acutely by augmenting spinal excitability using a potassium channel blocker (4-aminopyridine) or chronically with a long survival period (Brus-Ramer et al., 2009). Activity-dependent outgrowth likely overcomes this dependence, thereby enabling the ipsilateral control functions of motor cortex to be expressed after injury.

Brain electrical activity can also be augmented by motor training (Calautti and Baron, 2003; Tyc and Boyadjian, 2006; Rosenkranz et al., 2007). Forced used of the impaired limb by constraining the unimpaired limb [constraint-induced movement therapy (CIMT)] has been a particularly useful activity-based strategy for restoring motor function (Langhorne et al., 2009). Indeed, rats with pyramidotomy and constraint of the unimpaired forelimb showed improvement of ladder walking and increased CS axon outgrowth to the impaired side of the spinal cord compared with rats having constraint of the impaired forelimb (Maier et al., 2008). Humans with unilateral stroke also benefit from CIMT (Wolf et al., 2006), an effect accompanied by strengthening of CS connections (Wittenberg and Schaechter, 2009). These studies suggest that the CS system may mediate a significant proportion of the CIMT effect even though the treatment does not specifically target the CS system. Consistent with this view is that the rats subjected to CIMT (Maier et al., 2008) show a pattern of ipsilateral CST outgrowth that is remarkably similar to what we see with M1 (e.g., Fig. 5) and pyramid (Brus-Ramer et al., 2007) stimulation.

Whereas CIMT is appealing because of its efficacy, safety, and ease of use, electrical stimulation has several advantages. First, it can be targeted selectively at the CS system, increasing the strength and function of the particular motor system involved in many specific aspects of motor control. Second, treatment can be implemented in individuals who cannot participate in active physical therapy, such as patients with severe paralysis or young children. Finally, electrical stimulation may tap into biological processes that are not activated by physical therapy. Ideally, electrical stimulation and CIMT would be synergistic, allowing each to reinforce the other. Indeed, motor cortex stimulation can be used to augment the effect of practice on motor learning in healthy adults (Reis et al., 2009).

Several investigators have shown that motor cortex electrical stimulation can improve motor performance. These efforts have 
focused on supporting the injured hemisphere after stroke. In rats, cortical stimulation of the partially injured motor cortex combined with motor training significantly improved skilled reaching (Adkins et al., 2006, 2008). In monkeys, electrical stimulation improved hand motor function and expanded the cortical motor map when paired with rehabilitation training (Plautz et al., 2003). These studies indicate that cortical stimulation is effective in preclinical models. Human trials were prompted by these studies using implantable epidural stimulation (Levy et al., 2008). Although epidural motor cortex stimulation can be done safely (Levy et al., 2008), the phase III clinical trial did not show efficacy, possibly due to timing, location, or strength of stimulation, or the heterogeneity of the patients enrolled (Plow et al., 2009).

Motor cortex stimulation can be done noninvasively, using transcranial magnetic stimulation or transcranial direct current stimulation (Bolognini et al., 2009). These stimulation techniques alter the excitability of the corticospinal system (Bergmann et al., 2009; Perez and Cohen, 2009), and have already been used to safely improve motor function in humans with stroke (Fregni et al., 2005, 2006; Bolognini et al., 2009) or congenital hemiplegia (Kirton et al., 2008). Animal models of CST stimulation will be crucial to understanding the biological effects of stimulation and for maximizing efficacy. Our study demonstrates that electrical stimulation of the intact CS system after a complete unilateral lesion can help restore CS innervation to the affected side of the spinal cord and return fine motor control to previously impaired limbs.

\section{References}

Adkins DL, Campos P, Quach D, Borromeo M, Schallert K, Jones TA (2006) Epidural cortical stimulation enhances motor function after sensorimotor cortical infarcts in rats. Exp Neurol 200:356-370.

Adkins DL, Hsu JE, Jones TA (2008) Motor cortical stimulation promotes synaptic plasticity and behavioral improvements following sensorimotor cortex lesions. Exp Neurol 212:14-28.

Allred RP, Adkins DL, Woodlee MT, Husbands LC, Maldonado MA, Kane JR, Schallert T, Jones TA (2008) The vermicelli handling test: a simple quantitative measure of dexterous forepaw function in rats. J Neurosci Methods 170:229-244.

Bareyre FM, Kerschensteiner M, Raineteau O, Mettenleiter TC, Weinmann O, Schwab ME (2004) The injured spinal cord spontaneously forms a new intraspinal circuit in adult rats. Nat Neurosci 7:269-277.

Bergmann TO, Groppa S, Seeger M, Mölle M, Marshall L, Siebner HR (2009) Acute changes in motor cortical excitability during slow oscillatory and constant anodal transcranial direct current stimulation. J Neurophysiol 102:2303-2311.

Bolognini N, Pascual-Leone A, Fregni F (2009) Using non-invasive brain stimulation to augment motor training-induced plasticity. J Neuroeng Rehabil 6:8.

Bradbury EJ, Moon LD, Popat RJ, King VR, Bennett GS, Patel PN, Fawcett JW, McMahon SB (2002) Chondroitinase ABC promotes functional recovery after spinal cord injury. Nature 416:636-640.

Brösamle C, Schwab ME (1997) Cells of origin, course, and termination patterns of the ventral, uncrossed component of the mature rat corticospinal tract. J Comp Neurol 386:293-303.

Brus-Ramer M, Carmel JB, Chakrabarty S, Martin JH (2007) Electrical stimulation of spared corticospinal axons augments connections with ipsilateral spinal motor circuits after injury. J Neurosci 27:13793-13801.

Brus-Ramer M, Carmel JB, Martin JH (2009) Motor cortex bilateral motor representation depends on subcortical and interhemispheric interactions. J Neurosci 29:6196-6206.

Calautti C, Baron JC (2003) Functional neuroimaging studies of motor recovery after stroke in adults: a review. Stroke 34:1553-1566.

Dergham P, Ellezam B, Essagian C, Avedissian H, Lubell WD, McKerracher L (2002) Rho signaling pathway targeted to promote spinal cord repair. J Neurosci 22:6570-6577.

Fitts PM (1954) The information capacity of the human motor system in controlling the amplitude of movement. J Exp Psychol 47:381-391.
Fregni F, Boggio PS, Mansur CG, Wagner T, Ferreira MJ, Lima MC, Rigonatti SP, Marcolin MA, Freedman SD, Nitsche MA, Pascual-Leone A (2005) Transcranial direct current stimulation of the unaffected hemisphere in stroke patients. Neuroreport 16:1551-1555.

Fregni F, Boggio PS, Valle AC, Rocha RR, Duarte J, Ferreira MJ, Wagner T, Fecteau S, Rigonatti SP, Riberto M, Freedman SD, Pascual-Leone A (2006) A sham-controlled trial of a 5-day course of repetitive transcranial magnetic stimulation of the unaffected hemisphere in stroke patients. Stroke 37:2115-2122.

Freund P, Schmidlin E, Wannier T, Bloch J, Mir A, Schwab ME, Rouiller EM (2006) Nogo-A-specific antibody treatment enhances sprouting and functional recovery after cervical lesion in adult primates. Nat Med 12:790-792.

Friel KM, Martin JH (2007) Bilateral activity-dependent interactions in the developing corticospinal system. J Neurosci 27:11083-11090.

Friel KM, Drew T, Martin JH (2007) Differential activity-dependent development of corticospinal control of movement and final limb position during visually guided locomotion. J Neurophysiol 97:3396-3406.

Kirton A, Chen R, Friefeld S, Gunraj C, Pontigon AM, Deveber G (2008) Contralesional repetitive transcranial magnetic stimulation for chronic hemiparesis in subcortical paediatric stroke: a randomised trial. Lancet Neurol 7:507-513.

Kluver H, Barrera E (1953) A method for the combined staining of cells and fibers in the nervous system. J Neuropathol Exp Neurol 12:400-403.

Kuypers HGJM (1981) Anatomy of the descending pathways. In: Handbook of physiology (Brooks VB, ed), pp 345-422. Baltimore: Waverley.

Langhorne P, Coupar F, Pollock A (2009) Motor recovery after stroke: a systematic review. Lancet Neurol 8:741-754.

Levy R, Ruland S, Weinand M, Lowry D, Dafer R, Bakay R (2008) Cortical stimulation for the rehabilitation of patients with hemiparetic stroke: a multicenter feasibility study of safety and efficacy. J Neurosurg 108:707-714

Lindenberg R, Renga V, Zhu LL, Betzler F, Alsop D, Schlaug G (2010) Structural integrity of corticospinal motor fibers predicts motor impairment in chronic stroke. Neurology 74:280-287.

Lotze M, Sauseng P, Staudt M (2009) Functional relevance of ipsilateral motor activation in congenital hemiparesis as tested by fMRI-navigated TMS. Exp Neurol 217:440-443.

Maier IC, Baumann K, Thallmair M, Weinmann O, Scholl J, Schwab ME (2008) Constraint-induced movement therapy in the adult rat after unilateral corticospinal tract injury. J Neurosci 28:9386-9403.

Martin JH, Donarummo L, Hacking A (2000) Impairments in prehension produced by early postnatal sensory motor cortex activity blockade. J Neurophysiol 83:895-906.

Martin JH, Choy M, Pullman S, Meng Z (2004) Corticospinal system development depends on motor experience. J Neurosci 24:2122-2132.

Martin J, Friel K, Salimi I, Chakrabarty S (2009) Corticospinal development. In: Encyclopedia of neuroscience (Squire L, ed), pp 302-314. Oxford: Academic.

Metz GA, Whishaw IQ (2002) Cortical and subcortical lesions impair skilled walking in the ladder rung walking test: a new task to evaluate foreand hindlimb stepping, placing, and co-ordination. J Neurosci Methods 115:169-179.

Morris R, Cheunsuang O, Stewart A, Maxwell D (2004) Spinal dorsal horn neurone targets for nociceptive primary afferents: do single neurone morphological characteristics suggest how nociceptive information is processed at the spinal level. Brain Res Brain Res Rev 46:173-190.

Mouton PR, Gokhale AM, Ward NL, West MJ (2002) Stereological length estimation using spherical probes. J Microsc 206:54-64.

Perez MA, Cohen LG (2009) The corticospinal system and transcranial magnetic stimulation in stroke. Top Stroke Rehabil 16:254-269.

Plautz EJ, Barbay S, Frost SB, Friel KM, Dancause N, Zoubina EV, Stowe AM, Quaney BM, Nudo RJ (2003) Post-infarct cortical plasticity and behavioral recovery using concurrent cortical stimulation and rehabilitative training: a feasibility study in primates. Neurol Res 25:801-810.

Plow EB, Carey JR, Nudo RJ, Pascual-Leone A (2009) Invasive cortical stimulation to promote recovery of function after stroke: a critical appraisal. Stroke 40:1926-1931.

Porter R, Lemon RN (1993) Corticospinal function and voluntary movement. Oxford: Oxford UP.

Reis J, Schambra HM, Cohen LG, Buch ER, Fritsch B, Zarahn E, Celnik PA, Krakauer JW (2009) Noninvasive cortical stimulation enhances motor 
skill acquisition over multiple days through an effect on consolidation. Proc Natl Acad Sci USA 106:1590-1595.

Rosenkranz K, Kacar A, Rothwell JC (2007) Differential modulation of motor cortical plasticity and excitability in early and late phases of human motor learning. J Neurosci 27:12058-12066.

Rossignol S, Chau C, Brustein E, Belanger M, Barbeau H, Drew T (1996) Locomotor capacities after complete and partial lesions of the spinal cord. Acta Neurobiol Exp (Wars) 56:449-463.

Salimi I, Martin JH (2004) Rescuing transient corticospinal terminations and promoting growth with corticospinal stimulation in kittens. J Neurosci 24:4952-4961.

Schwab ME, Brösamle C (1997) Regeneration of lesioned corticospinal tract fibers in the adult rat spinal cord under experimental conditions. Spinal Cord 35:469-473.

Sukal TM, Ellis MD, Dewald JP (2007) Shoulder abduction-induced reductions in reaching work area following hemiparetic stroke: neuroscientific implications. Exp Brain Res 183:215-223.

Thomas B, Eyssen M, Peeters R, Molenaers G, Van Hecke P, De Cock P, Sunaert S (2005) Quantitative diffusion tensor imaging in cerebral palsy due to periventricular white matter injury. Brain 128:2562-2577.

Todd AJ (2002) Anatomy of primary afferents and projection neurones in the rat spinal dorsal horn with particular emphasis on substance $P$ and the neurokinin 1 receptor. Exp Physiol 87:245-249.
Tuszynski MH, Grill R, Jones LL, Brant A, Blesch A, Löw K, Lacroix S, Lu P (2003) NT-3 gene delivery elicits growth of chronically injured corticospinal axons and modestly improves functional deficits after chronic scar resection. Exp Neurol 181:47-56.

Tyc F, Boyadjian A (2006) Cortical plasticity and motor activity studied with transcranial magnetic stimulation. Rev Neurosci 17:469-495.

Weidner N, Ner A, Salimi N, Tuszynski MH (2001) Spontaneous corticospinal axonal plasticity and functional recovery after adult central nervous system injury. Proc Natl Acad Sci USA 98:3513-3518.

Werhahn KJ, Conforto AB, Kadom N, Hallett M, Cohen LG (2003) Contribution of the ipsilateral motor cortex to recovery after chronic stroke. Ann Neurol 54:464-472.

Whishaw IQ, Pellis SM, Gorny B, Kolb B, Tetzlaff W (1993) Proximal and distal impairments in rat forelimb use in reaching follow unilateral pyramidal tract lesions. Behav Brain Res 56:59-76.

Wittenberg GF, Schaechter JD (2009) The neural basis of constraintinduced movement therapy. Curr Opin Neurol 22:582-588.

Wolf SL, Winstein CJ, Miller JP, Taub E, Uswatte G, Morris D, Giuliani C, Light KE, Nichols-Larsen D, for the EXCITE Investigators (2006) Effect of constraint-induced movement therapy on upper extremity function 3-9 months after stroke: the EXCITE randomized clinical trial. JAMA 296:2095-2104. 